\title{
Neutropenia in Pediatric Infections
}

\author{
Aditya Kumar Gupta ${ }^{1}$ - Jagdish Prasad Meena ${ }^{1}$
}

Received: 2 July 2019 / Accepted: 2 July 2019 / Published online: 11 July 2019

(C) Dr. K C Chaudhuri Foundation 2019

Neutrophils play an important role in the innate immunity and account for majority of the circulating white blood cells (WBC). They are the prime responders to infections by toxin mediated killing of pathogens after phagocytosis. The half-life of neutrophils in circulation is approximately 7-12 h, and this can shorten during infective episodes due to their tissue migration. Neutropenia is often defined as the absolute neutrophil count $(\mathrm{ANC})<1500 /$ cumm but ideally this should be compared to age, sex and race matched healthy populations. Appropriate reference ranges are important to diagnose a child with neutropenia. Adult values of ANC are achieved by adolescence. For white children the lower limit of the ANC is 1000 /cumm below one year of age and 1500/cumm after infancy [1].

Causes of neutropenia include congenital causes like severe congenital neutropenia, cyclical neutropenia, inherited bone marrow failure syndromes, immunodeficiencies etc. and acquired causes like infections, drugs, autoimmunity, nutritional deficiencies, bone marrow failure or infiltration and splenic sequestration. In viral infections the decreased ANC may be due to direct viral infection of the hematopoietic progenitors, resulting in decreased proliferation and maturation, or to cytokine induced apoptosis of marrow cells by the stimulated host's immune system. Bacterial infections may result in neutropenia due to increased neutrophil utilisation and destruction, which on the blood film manifests as left-shift, with increased presence of band forms, and toxic granulation of the neutrophils [2].

The current issue of this journal has published an observational study by Mahajan et al. [3] focussing on the etiologies and outcomes of transient neutropenia in

Jagdish Prasad Meena drjpmeena@yahoo.com

1 Division of Pediatric Oncology, Department of Pediatrics, All India Institute of Medical Sciences, New Delhi 110029, India pediatric patients who were admitted after presenting to the emergency. The authors have highlighted that infections are an important cause of transient neutropenia in these patients with dengue, typhoid and malaria being the common etiologies. The length of hospital stay as well as the duration of neutropenia increased with the severity of neutropenia and there was an inverse relation of these to the age. The authors should be complimented for this study which shows that most of the episodes of transient infection induced neutropenia recovered without major complications.

There are however some lacunae that exist in this study. The children presenting to the emergency are likely to be more sick with organ dysfunction, which could be clinical or subclinical. Viral infections are a common cause of transient neutropenia and many children do not require admission [4]. The children presenting to the outpatient who could be neutropenic have not been included. Further, it would have been more informative to mention the total number of patients of a particular diagnosed infection who developed neutropenia during the study period. A comparison between outcomes of patients with and without neutropenia would be prudent. Whether underlying nutritional deficiencies and genetic polymorphisms could be responsible for some patients developing neutropenia after an infection when others do not, can be a question for further research.

It is likely that the children who presented to the emergency department could have received some medication. The authors fail to assess any relation between drugs administered (both before and during the study) and the transient neutropenia. The follow-up of these children has not been mentioned. A cross-sectional study will miss out on cases of recurrent neutropenia (e.g., if it was the first presentation of an infant with cyclical neutropenia).

A single cut-off of 1500 /cumm for defining neutropenia across all age groups and ethnicities is not appropriate, as the ANC may differ in these groups, although we do acknowledge that age, sex and ethnicity based ANC cutoff for the Indian population has not been defined. The 
same is evident from the data published in a recent multiethnic study involving 26,542 normal children below 6 y of age in which $10.2 \%$ of Indian children were found to have $\mathrm{ANC}<1500 /$ cumm [5].

It can be concluded that age, sex and ethinicity based data for hematological parameters need to be established for the Indian population and studies with larger sample size, involving normal as well as sick children are needed to establish the true prevalence and outcomes of transient neutropenia in children.

\section{Compliance with Ethical Standards}

Conflict of Interest None.

\section{References}

1. Boxer LA. How to approach neutropenia. Hematol Am Soc Hematol Educ Prog. 2012;2012:174-82.

2. Thomas AE, Simpson LA. A step-by-step approach to paediatric neutropenia. Pediatr Child Health. 2017;27:511-6.

3. Mahajan A, Kumar V, Sindhwani SP, Chhapola V. Clinical profile and short term outcome of children with neutropenia. Indian $\mathrm{J}$ Pediatr. 2019. https://doi.org/10.1007/s12098-019-03020-4.

4. Celkan T, Koc BS. Approach to the patient with neutropenia in childhood. Turk Pediatr Ars. 2015;50:136-44.

5. Denic S, Narchi S, Al Mekaini LA, et al. Prevelance of neutropenia in children by nationality. BMC Hematol. 2016;16:15.

Publisher's Note Springer Nature remains neutral with regard to jurisdictional claims in published maps and institutional affiliations. 